\title{
PERCEPCIONES DEL ALUMNADO DE GRADO DE EDUCACIÓN INFANTIL EN TORNO A LA CREACIÓN DE AUDIO Y VÍDEO PODCAST PARA LA ENSEÑANZA Y APRENDIZAJE DE LENGUAS EXTRANJERAS
}

\author{
STUDENT TEACHERS' PERCEPTIONS OF THE CREATION OF AUDIO AND \\ VIIDEO PODCAST FOR FOREIGN LANGUAGE TEACHING AND LEARNING
}

\author{
Gemma Tur Ferrer \\ gemma.tur@uib.es \\ Universidad de las Islas Baleares
}

\section{RESUMEN}

En este artículo se presenta una investigación sobre las percepciones del alumnado de los estudios Grado de Educación Infantil de la Universidad de las Islas Baleares en la sede de Ibiza, en relación al audio y vídeo podcast para la enseñanza y el aprendizaje de lenguas extranjeras. Los resultados muestran, en general, una actitud favorable hacia este tipo de recursos y actividades en educación. Las conclusiones permiten observar como este estudio discute algunos resultados de otro anterior, en el que surgían contradicciones en cuanto se analizaban aspectos concretos del impacto de la creación de podcasts para el aprendizaje de lenguas.

PALABRAS CLAVE: Audio podcast, vídeo podcast, formación docente inicial, enseñanzaaprendizaje de lenguas extranjeras.

\section{ABSTRACT}

This article presents a research project at the University of the Balearic Islands in the Ibiza centre, on student teachers' perceptions towards audio and vídeo podcast for language teaching and learning. Findings show a general positive attitude towards audio and vídeo podcast in education. Conclusions allow us to observe that this research refutes a previous one where some contradictions arose when referring to the concrete impact of podcast for language learning.

KEYWORDS: Audio podcast, vídeo podcast, pre-service teacher education, foreign language teaching and learning. 


\section{INTRODUCCIÓN}

En el marco de un proyecto amplio de introducción de la tecnología en los estudios de Grado de Educación Infantil (GEDI) en la sede de Ibiza de la Universidad de las Islas Baleares (UIB), se proponen dos actividades de creación de audio y vídeo podcast posteriores a las clásicas actividades de lengua de creación y corrección de textos escritos. Con estas actividades, se pretende trabajar además de la competencia en lengua escrita, las habilidades de pronunciación, ritmo y entonación propias de la competencia oral, entre otras competencias más allá de las lingüísticas. También se pretende la reflexión sobre las posibilidades de las Tecnologías de la Información y la Comunicación (TIC) para el aprendizaje, así como un posicionamiento del alumnado para su integración en su futura docencia. Como se puede observar en la figura 1, la propuesta tiene varias fases: primero, se escriben y corrigen los textos. Seguidamente, se diseñan y graban los artefactos de audio o vídeo. Finalmente, la actividad se completa publicando el podcast en los eportafolios del alumnado. Una selección de los podcasts creados en los cursos 2011-12 y 2012-13 se puede encontrar en el blog de actividades del alumnado que mantiene la profesora ${ }^{1}$.

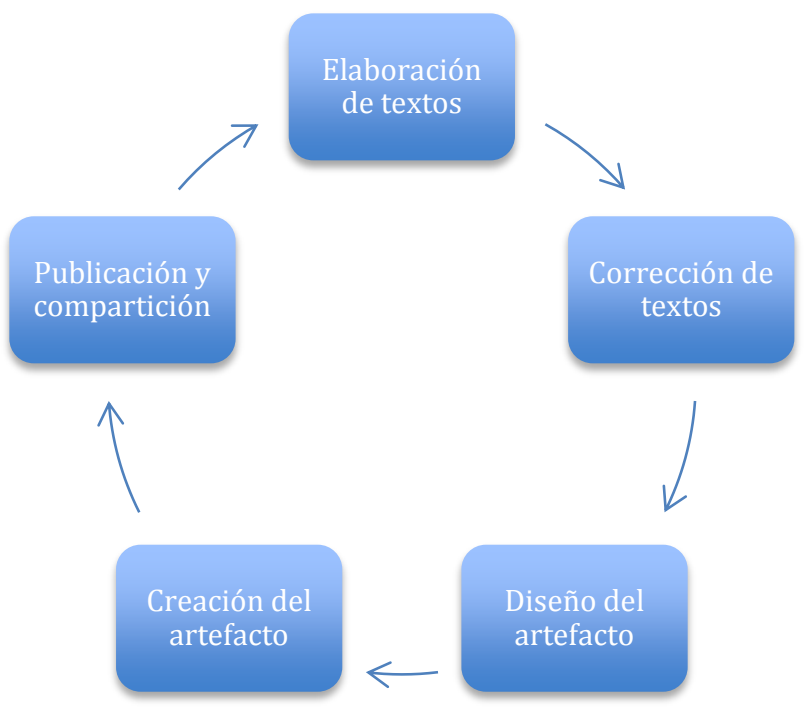

Figura 1. Actividades de podcast en las asignaturas de lengua extranjera de GEDI (UIB, Sede Ibiza). 


\section{AUDIO PODCAST EN LA EDUCACIÓN}

Las definiciones de podcast de Anderson (2007) y Redecker, Ala-Mutka, Bacigalupo, Ferrari y Punie (2009) son complementarias para definir el concepto que nos ocupa, ya que describen tanto su esencia como su funcionamiento, detalle que es clave para diferenciar el podcast de la creación de un archivo de audio.

Anderson $(2007,10)$ define los podcasts como grabaciones de audio, normalmente en formato MP3, que suelen ser de conferencias o entrevistas y que se pueden escuchar a través de un ordenador o tecnología móvil. Redecker $(2009,36)$ concreta que es la manera en la que una persona se mantiene actualizada con el contenido de audio más reciente. En resumen, como añade también Anderson (2007), el podcast es un servicio de actualización mediante RSS de las grabaciones de audio.

Como dice Anderson (2007) el nombre surge originariamente de iPod, con el que se conoce al célebre dispositivo portátil de Apple para reproducir archivos multimedia. Sin embargo, actualmente ya se ha extendido a la creación de archivos de sonido, en general, que pueden ser reproducidos por cualquier dispositivo móvil. Como otras herramientas de la Web 2.0, el podcast se ha prodigado mucho en educación, debido a sus múltiples posibilidades para la creación y difusión de contenido.

Los podcast tienen una gran potencialidad para el aprendizaje (Villalustre, 2013). Heilesen (2010) reflexiona sobre la oportunidad que ofrece la creación de podcast de una clase o una conferencia, dado que posibilita la audición tantas veces como se requiera, facilitando así la toma de notas. Además, en el caso de la creación de podcast, observa que sólo el hecho de tener que comunicar la información, aumenta considerablemente las posibilidades del aprendizaje. Para Nie, Armellini, Harrington, Barklamb y Randall (2010) el podcast tiene posibilidades para la flexibilización del currículum, la personalización de la enseñanza y la transformación de la institución. Aún así, conviene tener en cuenta que la innovación educativa con el uso de podcast no viene de la tecnología en sí misma sino de la integración curricular que se diseñe e implemente (Solano y Sánchez, 2010). La investigación de Trujillo (2011) sobre una experiencia en Educación Superior muestra la actitud positiva del alumnado hacia el podcast, enfatizando las posibilidades para la colaboración que implica su creación; no obstante, observa también limitaciones en torno a la percepción que tiene el propio alumnado para la mejora del aprendizaje.

En el caso concreto de las lenguas extranjeras, existen tres tipos de podcast, según Chacón y Clevia $(2011,43)$ : podcast auténtico, que suele crearse por nativos y sin propósito educativo; podcast del profesor, específico con contenidos objeto de aprendizaje; y, podcast del estudiante, creado por el alumnado para desarrollar habilidades de expresión y comprensión oral. 


\section{EL VÍDEO EN LA EDUCACIÓN}

Los vídeo podcasts o vídeocasts o vodcasts ${ }^{2}$ son archivos audiovisuales que se distribuyen en formato digital por Internet y que se pueden visionar desde un ordenador o aparato móvil, según la definición de McGarr (2009) citada por Kay y Kletskin $(2012,619)$. Para Bartolomé (2008) las posibilidades para la creación, edición y publicación de vídeo constituyen una de las características más definitorias de la Web 2.0. Este hecho ha supuesto que vuelva a resurgir con fuerza como recurso educativo. De hecho, su uso se ha incrementado en los últimos tiempos también en la Educación Superior, como recogen Kay y Kletskin (2012). Puesto que el vídeo tenía presencia antes de la Web 2.0, podemos encontrar un marco teórico sólido al respecto. Cabero $(2006,130)$ hace un resumen de las posibilidades y limitaciones del vídeo, que mostramos resumidamente a continuación:

\begin{tabular}{|c|c|}
\hline POSIBILIDADES & LIMITACIONES \\
\hline $\begin{array}{l}\text { - El programa puede visualizarse un } \\
\text { número indefinido de veces } \\
\text { - Posibilidad de introducir mensajes de } \\
\text { otros medios como la televisión y el } \\
\text { cine } \\
\text { - } \quad \text { La disminución progresiva del coste de } \\
\text { los equipos } \\
\text { - } \quad \text { Posibilidades de utilizarse en diferentes } \\
\text { niveles educativos } \\
\text { - } \quad \text { Facilidad progresiva de los aparatoso } \\
\text { de grabación y reproducción de vídeo } \\
\text { - Diversidad de funciones por las que se } \\
\text { puede usar en educación } \\
\text { - Favorece un uso activo } \\
\text { - Puede ser fácilmente incorporado en } \\
\text { un conjunto de materiales multimedia y } \\
\text { en red. }\end{array}$ & $\begin{array}{l}\text { - Su producción por parte de profesorado } \\
\text { y alumnado, requiere de formación } \\
\text { específica } \\
\text { - } \quad \text { Se necesita una mínima dotación } \\
\text { instrumental } \\
\text { - } \quad \text { Es preciso tener una mínima dotación } \\
\text { instrumental } \\
\text { - } \quad \text { Puede favorecer la pasividad } \\
\text { - } \quad \text { Limitaciones del “copyright" que no } \\
\text { suelen considerarse en los centros } \\
\text { educativos. }\end{array}$ \\
\hline
\end{tabular}

Tabla 1. Posibilidades y limitaciones para el uso del vídeo en la enseñanza. Cabero $(2006,130)$

Cabero $(2006,132)$ distingue ocho usos didácticos del vídeo que citamos a continuación: transmisor de conocimiento, instrumento motivador, instrumento de conocimiento por el alumnado, instrumento de evaluación, formación del profesorado en estrategias didácticas,

\footnotetext{
${ }^{2}$ Otras nomenclaturas que recoge Kay $(2012,821)$ son audiographs, webcasts o vídeo streams
} 
para la formación del profesorado en contenido, herramienta de investigación psicodidáctica, por la investigación de procesos desarrollados en el laboratorio, instrumento de comunicación y alfabetización icónica / medio de comunicación del alumnado, e instrumento para el análisis de los medios. Consideramos que algunos de estos usos han adquirido especial protagonismo con los servicios de vídeo que la Web 2.0 ha favorecido.

\subsection{Los servicios de vídeo podcast en la educación}

Siguiendo a Kay (2012), la investigación en torno a los servicios de vídeo podcast comenzó tímidamente el año 2002, época en la que su uso era muy limitado en educación. Dos fueron los factores que fomentaron su uso en educación y que, en consecuencia, promovieron también la investigación: el nacimiento de Youtube en 2005 y el desarrollo de la banda ancha entre 2006 y 2010.

Para Kay $(2012,821)$ los vídeo podcasts se pueden clasificar de la siguiente manera:

- Propósito. En esta tipología, hay cuatro categorías. La primera, las lecciones, son unidades íntegras de contenido que el alumnado puede visualizar antes o después de la clase. La segunda, vídeos mejorados, elaborados a partir de otros recursos, como presentaciones con audio, etc. La categoría de suplementarios, constituida por vídeos con material complementario que puede consistir en ayuda administrativa, demostraciones, resúmenes o material adicional para ampliar o profundizar. Y finalmente, la categoría de ejemplos, consistente en vídeos con explicaciones sobre problemas específicos que el alumnado debe saber resolver, especialmente, en áreas de ciencias.

- Segmentación, con dos tipologías: vídeos segmentados y no segmentados. Los primeros son los que se pueden visualizar completamente desde el principio hasta el final, y los segundos, lo que están divididos en fragmentos, permitiendo la búsqueda y selección de segmentos concretos, según las necesidades.

- Estrategia pedagógica, formada por tres subgrupos: visualización receptiva, resolución de problemas y creación de vídeo podcasts. En el primer acercamiento pedagógico, se entiende que el alumnado debe ver los vídeos de una manera pasiva. Es el tratamiento pedagógico más común, según este autor. En el segundo, se trata de ayudar al alumnado en el aprendizaje de la resolución de problemas. En la tercera tipología, se trata de la planificación y creación de sus propios vídeos. El alumnado aprende investigando, colaborando, buscando y, finalmente, creando vídeos de tipo académico, lo cual no es un tratamiento pedagógico muy común, según Kay (2012).

- Enfoque académico, con dos únicas categorías: prácticos y conceptuales. Según este autor, la mayoría de vídeos que se crean son de la primera categoría, que se caracterizan por ser de corta duración o segmentados sobre ejemplos prácticos o problemas específicos. Los segundos suelen ser más largos, tratan "higher level concepts" (Kay, 2012, 822) y también suelen ser segmentados. 
La investigación reciente en torno al vídeo, gira especialmente en relación a la influencia que los servicios de podcast de vídeo están teniendo en este medio, y en algunos de sus usos, especialmente, los relacionados con su consumo y creación por parte de alumnado y profesorado. Investigaciones como la de Schreiber, Fukuta y Gordon (2010) concluyen resultados contradictorios: el alumnado valora las posibilidades del vídeo podcast para la revisión y, sin embargo, también lo considera menos motivador que una clase presencial. Kay y Kletskin $(2012,620)$ señalan que los servicios de vídeo podcast tienen implicaciones positivas para el alumnado, tanto a nivel de actitud, como de procedimientos y resultados del aprendizaje. Así por ejemplo, indican a partir de resultados de investigación que: son entretenidos para visionar, motivadores, útiles y efectivos para mejorar el aprendizaje, útiles para la posibilidad de controlar su uso y ritmo de escucha, que promueven la autonomía del aprendizaje, aumentan la autorreflexión y que son eficientes para la preparación de exámenes, y para la mejora de los resultados y habilidades. Finalmente, argumentan que los vídeo podcasts no sólo son útiles para la exposición de contenidos sino que también lo son para la resolución de problemas de naturaleza procedimental.

Kay (2012) documenta los beneficios y desafíos que el uso del vídeo puede comportar. Entre los primeros, observa los siguientes:

- Razones para usar el vídeo podcast: la mejora del aprendizaje gracias al aumento de las posibilidades para revisar contenidos antes y después de las sesiones presenciales; el control sobre el aprendizaje que implica poder escuchar sólo aquella información que se busca; y la compensación en caso de haberse perdido sesiones de clase.

- Actitudes afectivas positivas hacia los vídeos. La investigación realizada en torno a las percepciones de los alumnos destaca los siguientes aspectos: entretenidos de visionar, satisfactorios, motivadores, y que ayudan a reducir la ansiedad.

- Actitudes cognitivas positivas hacia los vídeos. En este caso, la autora enumera y observa los siguientes comentarios del alumnado: son útiles para mejorar el aprendizaje, y en especial, en la creación de vídeos, ya que declaran una mayor habilidad para el análisis, comunicación, cooperación, creatividad y la tecnología.

- Impacto positivo en el comportamiento del alumnado. En este caso, se mejoran aspectos como los hábitos de estudio o la asistencia a clase.

- Impacto positivo en los resultados del aprendizaje, ya que la investigación valida la relación entre el alumnado que visiona vídeos y mejores resultados en pruebas tipo test.

Los retos que hay que superar, basándonos en esta misma autora (Kay, 2012), son los siguientes:

- Las razones para no usarlos: problemas técnicos, preferencias por la clásica lección presencial, la falta de tiempo e incluso el desconocimiento de su existencia. 
- Las actitudes negativas de tipo cognitivo, de las cuales se observa la preferencia del vídeo sólo como material complementario y cómo, a su vez, éstos se observan como repetitivos.

- El impacto negativo en el comportamiento del alumnado. En este caso, Kay (2012) recoge algunas investigaciones que observan una menor asistencia a clase en caso de visionar vídeos. También se observan más dificultades para el seguimiento de vídeos que para el seguimiento de clases tradicionales.

- Algunas investigaciones han observado escasas mejoras en el aprendizaje aunque sin determinar las razones.

Otras investigaciones destacan algunos aspectos más a considerar en torno al vídeo podacast. Así Özdener y Güngör (2010) observan que no hay diferencias entre la calidad de los contenidos de los vídeos publicados en red y los que se entregan en formato CD o DVD; en cambio, sí se observan diferencias en la calidad del diseño del proyecto y de la presentación. La investigación de Kazlauskas y Robinson (2012) destaca que el alumnado con poco tiempo para el estudio es el que menos lo usa. Tanto esta investigación como la de Copley (2013) añaden una nueva variable de estudio, como que la mayoría no aprovecha sus posibilidades para el aprendizaje ubicuo, separando claramente entre el tiempo de estudio y el tiempo de uso de los dispositivos móviles para el uso recreativo. De hecho, Evans (2008) destaca la escasa explotación que hace el alumnado del podcast para la multi-tarea.

Salinas (2012) hace hincapié en otro aspecto del vídeo podcast: los centros, profesorado y alumnado como emisores y no sólo como receptores. Para este autor, el vídeo puede tener un carácter bidireccional, aunque para aprovechar esta capacidad de emisión, no es suficiente el acceso a las herramientas ni un "uso intensivo" $(2012,19)$, sino que también es necesario que se domine el lenguaje. $Y$ a la vez, esta capacidad creativa para comunicar también dará a los alumnos la capacidad de recibir críticamente los mensajes de los medios. Es decir, el vídeo es un recurso útil tanto como medio como contenido para llegar, en definitiva, a la utilización cotidiana del medio.

Según Salinas (2012) la creación de vídeo, se integrará en procesos de aprendizaje colaborativo, permitiendo la colaboración en el desarrollo del proyecto. En esta línea, la investigación de Zahn, Krauskopf, Hesse y Pea (2012) muestra evidencias de la mejora del aprendizaje en caso de trabajo colaborativo en torno al vídeo. Siguiendo a Salinas (2012), aunque la producción de vídeo en la educación no llegue a una gran calidad técnica, por falta de recursos y porque el profesorado no es técnico de imagen y sonido, lo más deseable es que el vídeo se integre, progresivamente, en el currículo y el aula, en procesos de enseñanza-aprendizaje más centrados en los medios didácticos y la actividad del alumnado, que en el libro de texto. Igualmente, fomenta el rol del profesorado como emisor de contenido.

Finalmente, debe destacarse la investigación que trata el tema del vídeo podcast para el fomento de la reflexión y el razonamiento del alumnado. Para Frydenberg (2008) la creación de vídeo podcast requiere habilidades tecnológicas y cognitivas. Cheng y Chau (2009) 
plantean dos preguntas de investigación que tienen que ver con las posibilidades del vídeo para fomentar la reflexión y las consideraciones del alumnado sobre si esto es una actividad relevante para su aprendizaje. Los datos confirman la relación positiva entre la creación de vídeo y el apoyo a la reflexión y, por ello, también se acaban haciendo consideraciones pedagógicas para su uso. Fadde, Aud y Gilbert (2009) analizan las percepciones del alumnado en torno a la creación de vídeos para la reflexión. El análisis evidencia el nerviosismo ante la grabación, y la posterior valoración positiva del trabajo por parte del alumnado como actividad de feedback y de apoyo al análisis reflexivo.

\section{DESCRIPCIÓN DEL ESTUDIO}

Se trata de un estudio en relación a las percepciones del alumnado que realizan estas dos actividades de audio y vídeo podcast en las dos asignaturas de inglés. El total del grupo investigado está formado por 63 alumnos y alumnas (26 corresponden al curso 2011-12 y 37 al curso 2012-13), y la técnica de recogida de datos se basa en un cuestionario que se contesta anónimamente, al final del semestre, después de haber realizado idénticas actividades. El cuestionario es cerrado, con 12 preguntas cuyas respuestas, mayoritariamente, se han de elegir entre cuatro adverbios de cantidad organizados en una escala gradual. Sólo algunas preguntas son abiertas o presentan otro tipo de escala.

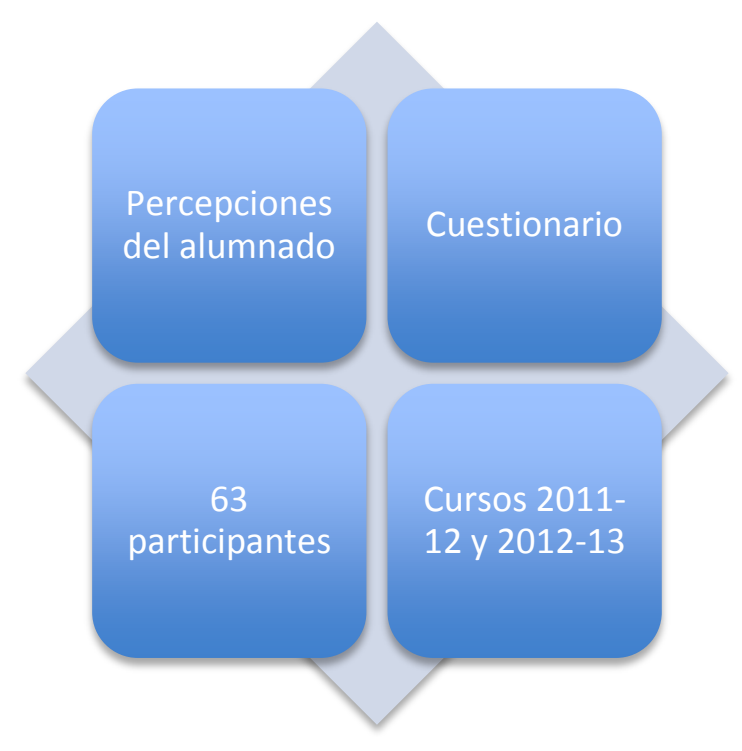

Figura 2. Descripción del estudio 


\section{RESULTADOS}

Los resultados de las preguntas seleccionadas se muestran en los siguientes gráficos de columnas:

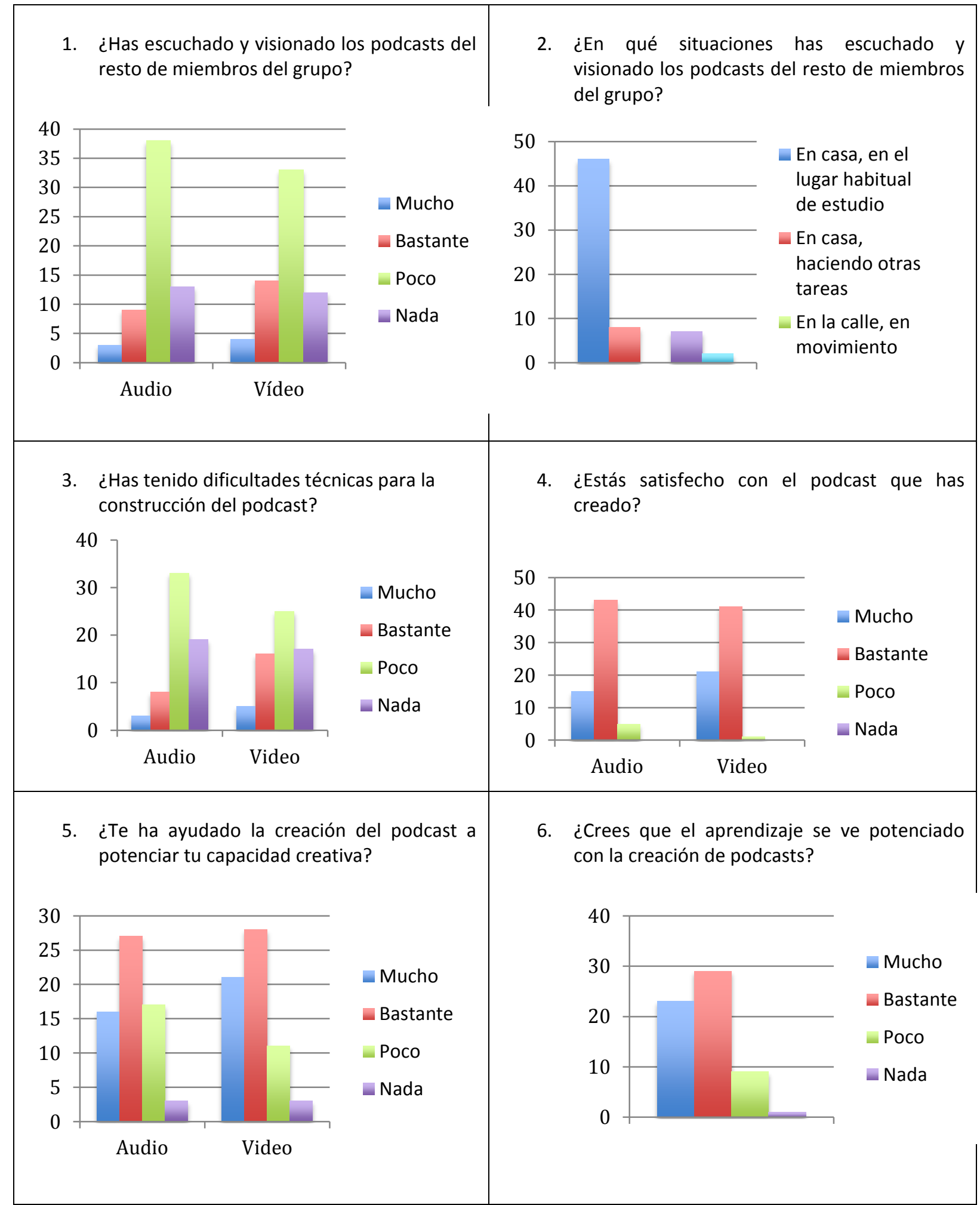




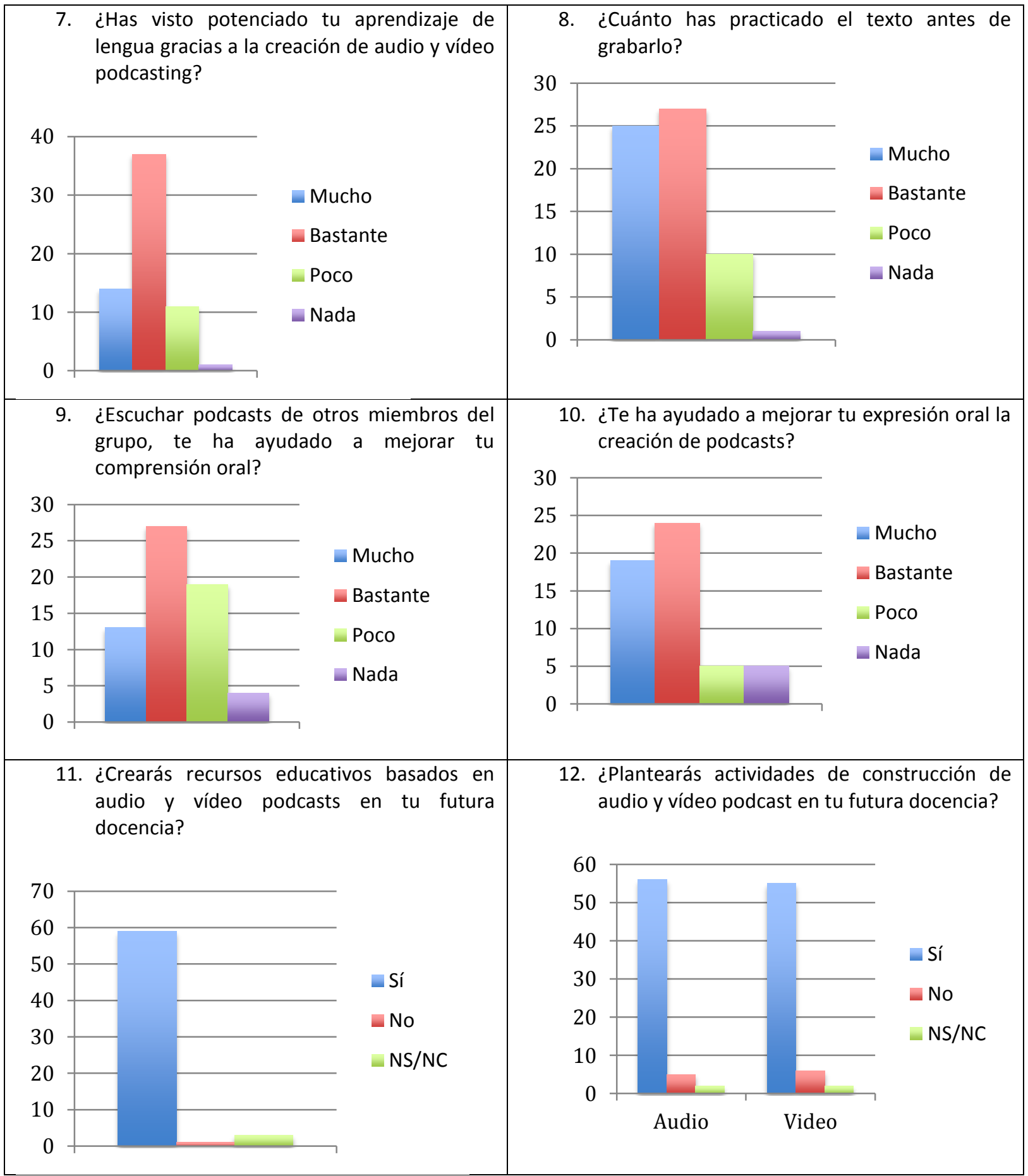

Tabla 2. Resultados del cuestionario 


\section{DISCUSIÓN}

En las preguntas más generales, se observa una satisfacción global del alumnado por su producción y percepciones positivas en relación al impacto sobre el propio aprendizaje. El alumnado está mayoritariamente muy o bastante satisfecho con su producto (pregunta 4) y considera que la creación del artefacto le ha ayudado a desarrollar su propia creatividad, especialmente, en la creación del vídeo (pregunta 5). Además, la mayoría del alumnado cree que esta actividad potencia el aprendizaje (pregunta 6) y, concretamente, también tiene percepciones positivas en cuanto al aprendizaje de lenguas (pregunta 7), probablemente, además por la oportunidad que ha tenido de practicar el texto antes de grabarlo (pregunta 8). La tendencia es similar en el caso de las preguntas que pretenden concretar en qué habilidad lingüística ha tenido mayor impacto (preguntas 9 y 10). En ambos casos se puede observar una mayoría de respuestas de "bastante", aunque con una ligera diferencia. En la pregunta 9, hay una cantidad importante de respuestas en el nivel "poco", lo cual es coherente con la pregunta 1, puesto que si el alumnado admite, mayoritariamente, haber escuchado "poco" los artefactos de sus compañeros, es normal que consideren que el impacto en su comprensión oral de la lengua extranjera también sea "poco". Destaca, en cambio, que en la pregunta 10 (impacto en la expresión oral) las respuestas en los niveles "mucho" y "bastante" suponen el $68 \%$ de total de respuestas, lo cual es coherente con la con las respuestas a la pregunta 8 -que busca saber si el alumnado, gracias a la actividad, se ha visto obligado a trabajar más intensamente el texto a grabar- que obtiene una mayoría de respuestas también entre los niveles "mucho" y "bastante" (83\%). También parece que estas respuestas son coherentes con las de la pregunta 7 (referida al impacto en el aprendizaje de lenguas). Dada la actitud positiva reflejada por el alumnado a partir de la experiencia de aprendizaje es coherente el resultado en las preguntas 11 y 12 . Así pues, el alumnado proyecta en su futura docencia la integración de la tecnología para la creación de audio y vídeo podcast. Prácticamente todo el grupo parece estar dispuesto para la creación de recursos didácticos basados en audio y vídeo podcast (pregunta 11) y también una gran parte del grupo, aunque no todo, está dispuesto a plantear actividades de creación de este tipo de objetos multimedia en un futuro a su alumnado (pregunta 12). La diferencia en las respuestas negativas entre audio y vídeo de la pregunta 12 parece denotar un ligero mayor rechazo en vídeo que no podemos explicar. Una investigación más profunda podría intentar averiguar si se debe al uso de su propia imagen o una mayor dificultad técnica. Tanto la creación del audio como el vídeo ha presentado pocas dificultades técnicas al alumnado, aunque en el caso del vídeo, aumenta el numero de estudiantes que afirman haber tenido "bastante" dificultad. Finalmente, otro aspecto que podría llevar a reflexiones interesantes estaría en que el alumnado, mayoritariamente, escucha y visualiza estos trabajos multimedia en situaciones de aprendizaje clásicas, siendo una minoría los que aprovechan otros momentos, recurriendo a dispositivos móviles para realizar esta actividad. Las respuestas abiertas a esta pregunta número dos muestran también hábitos de visionado en grupo. 


\section{CONCLUSIÓN}

Los datos conseguidos en esta nueva edición de la investigación son interesantes porque permiten confirmar algunas de las conclusiones a las que se pudieron llegar con una muestra más reducida. Pero, sobre todo, esta investigación es relevante porque abre nuevos interrogantes al contradecir algunos de los resultados anteriores en torno a las habilidades concretas del aprendizaje de lenguas extranjeras que se ven mejoradas gracias a la creación de audio y vídeo podcast.

Así pues, se confirman los resultados previos sobre las percepciones positivas del alumnado en torno al audio y vídeo podcast y sus expectativas para su futura docencia (Tur, 2013). Además, estos resultados sobre las implicaciones positivas del impacto en el aprendizaje, van en la línea de las investigaciones de Trujillo (2011), Kay (2012) y Kay y Kletskin (2012), así como la expresión de emociones positivas en relación al podcast, también en la línea de la investigación de Kay (2012).

Sin embargo, los resultados que permiten valorar el impacto observado en relación a la expresión oral en lengua extranjera, y en menor medida, a la comprensión oral, son contradictorios con la investigación anterior, en la que el alumnado no reconocía impacto alguno ni en el desarrollo de su competencia oral ni escrita en lengua extranjera (Tur, 2013). Y por lo tanto, estos nuevos resultados van en la línea de los obtenidos por Chacón y Clevia (2011), que sí concluyen el impacto en el desarrollo de las habilidades de comprensión y expresión oral del alumnado. Esto abre una interesante nueva línea futura de investigación en la que se podría profundizar en aspectos más concretos sobre el aprendizaje de lenguas extranjeras.

Las posibles resistencias a este tipo de actividades parece que no pueden basarse en dificultades técnicas porque la mayor parte del grupo responde que ha tenido pocas, lo que no coincide con las conclusiones de la investigación de Kay (2012) y de Tam (2012). La referencia a visionados en grupo va en la línea de las conclusiones de la investigación de Zahn, Krauskopf, Hesse y Pea (2012) que valoran las posibilidades del podcast para fomentar el trabajo colaborativo. Con respecto al escaso aprovechamiento de contextos de aprendizaje más informales entre el alumnado, se observa algo similar a lo contemplado por Kazlauskas y Robinson (2012) y Copley (2013) que concluyen que los podcasts aún no se explotan maximizando las posibilidades para el aprendizaje ubicuo. Estos resultados también van en la línea de los de Evans (2008) que no observa actividad multi-tarea del alumnado durante la audición de los podcast. Finalmente, las respuestas del alumnado sobre sus perspectivas de uso de la tecnología en su futura docencia, evidencian una actitud positiva mayoritaria. Aún así, de los datos obtenidos, no se puede analizar la argumentación pedagógica en la que el alumnado basa esta actitud positiva, ni si se observa el valor de la creación de este tipo de recursos como recursos educativos que van a permitir la progresiva independencia docente del libro de texto, en la línea de las aportaciones de Salinas (2012). 


\section{REFERENCIAS}

ANDERSON, P. (2007). What is web 2.0?: Ideas, technologies and implications for education. JISC Bristol, UK. Recuperado 25 de Julio 2013, de http://www.jisc.ac.uk/media/documents/techwatch/tsw0701b.pdf

BARTOLOMÉ, A. (2008). Web 2.0 and new learning paradigms. ELearning papers, no. 8. Recuperado de http://www.Elearningeuropa.Info/files/media/media15529.pdf

CABERO, J. (2006). El vídeo en la enseñanza y formación. En J. Cabero. (coord.) Nuevas Tecnologías aplicadas a la educación. Madrid; México: McGraw-Hill.

CHENG, G., y CHAU, J. (2009). Digital vídeo for fostering self-reflection in an eportfolio environment. Learning, Media and Technology, 34(4), 337-350. doi:10.1080/17439880903338614

COPLEY, J. (2013). Audio and vídeo podcasts of lectures for campus-based students: production and evaluation of students use. Innvoations in Education and Teaching International, 44 (4), 387-399. doi: doi:10.1080/14703290701602805

EVANS, C. (2008). The effectiveness of m-learning in the form of podcast revision lectures in higher education. Computers \& Education, 50, 491-498. doi: http://dx.doi.org/10.1016/j.compedu.2007.09.016

FADDE, P. J., AUD, S., y GILBERT, S. (2009). Incorporating a vídeo-editing activity in a reflective teaching course for preservice teachers. Action in Teacher Education, 31(1), 75-86. Recuperado de http://peterfadde.com/Research/ATE-VídeoEditActivity.pdf

FRYDENBERG, M. (2008). Principles and Pedagogy: the two Ps of Podcasting in the Information Technology Classroom. Information Systems Education Journal, 6 (6). Recuperado de http://proc.isecon.org/2006/3354/ISECON.2006. Frydenberg.pdf

HEILESEN, S. B. (2010). What is the academic efficacy of podcasting? Computers \& Education, 55(3), 1063-1068. doi:10.1016/j.compedu.2010.05.002

KAY, R. (2012). Exploring the use of vídeo podcasts in education: A comprehensive review of the literature. Computers in Human Behavior, 28, 820-831. doi:10.1016/j.chb.2012.01.011

KAY, R. y KLETSKIN, I. (2012). Evaluating the use of problem-based vídeo podcasts to teach mathematics in higher education. Computers \& Education, 59, 619-627. doi:10.1016/j.compedu.2012.03.007

KAZLAUSKAS, A., y ROBINSON, K. (2012). Podcasts are not for everyone. British Journal of Educational Technology, 43(2), 321-330. doi:10.1111/j.1467-8535.2010.01164.x

NIE, M., ARMELLINI, A., HARRINGTON, S., BARKLAMB, K., y RANDALL, R. (2010). The role of podcasting in effective curriculum renewal. Research in Learning Technology, 18, 2. doi: 10.1080/09687769.2010.492849

ÖZDENER, N., y GÜNGÖR, Y. (2010). Effects of vídeo podcast technology on peer learning and 
project quality. Procedia-Social and Behavioral Sciences, 2(2), 2217-2221. doi: 10.1016/j.sbspro.2010.03.311

REDECKER, C., ALA-MUTKA, K., BACIGALUPO, M., FERRARI, A., y PUNIE, Y. (2009). Learning 2.0: The impact of web 2.0 innovations on education and training in europe. Final Report. European Commission-Joint Research Center-Institute for Prospective Technological Studies, Seville. Recuperado 25 de Julio de 2013, de http://ipts.jrc.ec.europa.eu/publications/pub.cfm?id=2899

SALINAS, J. (2012). 'I uploaded a@ youtube vídeo':?' Una nueva perspectiva de la televisión educativa? EDMETIC, Revista De Educación Mediática Y TIC, 1(1), 7-28. Recuperado de http://www.edmetic.es/Documentos/Vol1Num1-2012/Jesus_Salinas Ibanez.pdf

SCHREIBER, B., FUKUTA, J. y GORDON, F. (2010). Live lectura versus vídeo podcast in undergraduate medical education: a randomised controlled trial. BMC Medical Education, 10. Recuperado de http://www.biomedcentral.com/1472-6920/10/68

SOLANO, I. y SÁNCHEZ, M. (2010). Aprendiendo en cualquier lugar: el podcast educativo. Pixel Bit. Revista de Medios y Educación, 36, 125-139. Recuperado de http://www.sav.us.es/pixelbit/pixelbit/articulos/n36/10.pdf

TAM, C. (2012). The effectiveness of educational podcasts for teaching music and visual arts in higher education. Research in Learning Technology, 20, 1. doi: I: 10.3402/rlt.v20i0.14919

TRUJILLO, J. M. (2011). Comunicación, innovación, educación y gestión del conocimiento en torno al uso del podcast en la educación superior. RUSC. Revista De Universidad Y Sociedad Del Conocimiento, 8(2). doi: 10.7238/rusc.v8i2.1047

TUR, G. (2013). Audio y vídeo podcast para el aprendizaje de lenguas extranjeras en la formación docente inicial. IV Jornadas Internacionales de Campus Virtuales, Palma 14 y 15 de febrero 2013. Recuperado de http://campusvirtuales2013.uib.es/docs/161.pdf

VILLALUSTRE, L. (2013). Narraciones digitales mediante podcast en plataformas virtuales. IV Jornadas Internacionales de Campus Virtuales, Palma 14 y 15 de febrero 2013. Recuperado de http://campusvirtuales2013.uib.es/docs/111.pdf

ZAHN, C., KRAUSKOPF, K., HESSE, F. W., y PEA, R. (2012). How to improve collaborative learning with vídeo tools in the classroom? Social vs. Cognitive guidance for student teams. International Journal of Computer-Supported Collaborative Learning, 7, 259-284. doi:10.1007/s11412-012-9145-0

\section{Para citar este artículo:}

TUR, G. (2013). Percepciones del alumnado de grado de educación infantil en torno a la creación de audio y vídeo podcast para la enseñanza y aprendizaje de lenguas extranjeras. EDUTEC, Revista Electrónica de Tecnología Educativa, 46. Recuperado el dd/mm/aa de http://edutec.rediris.es/Revelec2/Revelec46/percepcion alumnado grado educacion infantil podc ast lenguas estrangeras.html 